\title{
Inherited bone marrow failure syndromes in 2012
}

\author{
Hirotoshi Sakaguchi · Koji Nakanishi • \\ Seiji Kojima
}

Received: 11 October 2012/Revised: 7 December 2012/ Accepted: 10 December 2012/Published online: 28 December 2012

(C) The Japanese Society of Hematology 2012

\begin{abstract}
Inherited bone marrow failure syndromes (CBMFS) are a heterogeneous group of genetic disorders characterized by bone marrow failure, congenital anomalies, and an increased risk of malignant disease. The representative diseases with trilineage involvement are Fanconi anemia and dyskeratosis congenita, while the disease with the single lineage cytopenia is DiamondBlackfan anemia. Recent advances in our understanding of these diseases have come from the identification of genetic lesions responsible for the disease and their pathways. Although recent studies have identified many causative genes, mutations of these genes have only been found in less than half of the patients. Next-generation sequencing technologies may reveal new causative genes in these patients. Also, induced pluripotent stem cells derived from patients with CBMFS will be useful to study the pathophysiology of the diseases. The only long-term curative treatment for bone marrow failure in patients with inherited bone marrow failure syndromes is allogeneic hematopoietic stem cell transplantation, although this procedure has a risk of severe adverse effects. Multicenter prospective studies are warranted to establish appropriate conditioning regimens aimed at reducing transplant-related mortality.
\end{abstract}

Keywords Inherited bone marrow failure syndrome . Fanconi anemia $\cdot$ Diamond-Blackfan anemia . Dyskeratosis congenita

H. Sakaguchi · K. Nakanishi · S. Kojima ( () Department of Pediatrics,

Nagoya University Graduate School of Medicine, 65 Tsurumai-cho, Showa-ku, Nagoya 466-8550, Japan e-mail: kojimas@med.nagoya-u.ac.jp

\section{Introduction}

Inherited bone marrow failure syndromes (IBMFS) are a heterogeneous group of genetic disorders characterized by bone marrow failure, congenital anomalies, and increased risk of malignant disease. Such bone marrow failure may affect all three hematopoietic cell lineages or single cell lineages individually. Diseases characterized by trilineage involvement include Fanconi anemia and dyskeratosis congenita, while Diamond-Blackfan anemia results in single-lineage cytopenia. Recent advances in our understanding of these diseases have arisen from the identification of genetic lesions responsible for such diseases and their pathogenic pathways. These investigations have further clarified both normal and pathological hematopoiesis. In this current review, we describe recent insights into three IBMFS: Fanconi anemia, Diamond-Blackfan anemia, and dyskeratosis congenita.

\section{Fanconi anemia}

Fanconi anemia (FA) is a rare autosomal recessive disease characterized by congenital abnormalities, progressive bone marrow failure, and cancer susceptibility. FA, which has an incidence of less than 10 per million live births, is the most frequent inherited cause of aplastic anemia [1]. FA is a genetically heterogeneous disease defined by complementation groups. To date, 15 genes have been identified as playing a causative in FA and these genes, FANCA to FANCP, have been cloned [2]. Children with FA often develop aplastic anemia during the first decade of life, with death often resulting from complications of bone marrow failure, such as severe infection or bleeding. FA 
patients also develop clonal chromosomal abnormalities in bone-marrow progenitor cells, such as monosomy 7, which are associated with myelodysplastic syndrome (MDS) and acute myeloblastic leukemia (AML) [3]. The gene FANCD1, which is responsible for complementation group FA-D1, is identical to the hereditary breast cancer susceptibility gene, BRCA2, and has been reported as affected in $3 \%$ of patients with Fanconi anemia. As compared with children from other FA groups, more severe phenotypes are seen in FA-D1 patients, such as co-occurrence of multiple anomalies, development of multiple malignancies with earlier onset, and increased incidence of leukemia and solid tumors [4], including Wilms tumor, neuroblastoma, and brain tumors.

While the treatment of choice for FA patients remains allogeneic stem cell transplantation (SCT) from an HLAmatched sibling or unrelated donor, older patients may develop squamous-cell carcinomas (SCCs) of the head and neck or gynecological system. In particular, some studies have demonstrated there is a high incidence of SCC, such as esophageal cancer, in FA patients who have received SCT. The age-specific hazard of SCC has been shown to be 4.4-fold higher in patients who receive transplants and, in addition, SCCs occurred at significantly younger ages in the transplant group [5]. Thus, further investigations of the complete care of FA patients need to be undertaken.

Complementation groups and genes of FA

Since cells derived from FA patients are hypersensitive to DNA interstrand cross-linking (ICL) agents, such as diepoxybutane (DEB), mitomycin C (MMC), and cisplatin, it is expected that FA genes are involved in ICL repair. In 1993, FANCC was the first FA gene to be cloned by expression cloning [6, 7]. Subsequently, 15 other genes have been cloned (Table 1). At present, the FANC genes, which range from FANC"A" to FANC "P", and the FA pathway have been shown to resolve ICLs encountered during DNA replication. There are three primary groups of FA proteins, which include the FA core complex, the ID (FANCD2/I) complex, and the BRCA complex (Fig. 1). In these groups, there are eight FA proteins (FANCA/B/C/E/F/G/L/M) that form a multi-subunit ubiquitin E3 ligase complex, the FA core complex, which activates the monoubiquitination of the ID complex after genotoxic stress, such as ICL, or during the $\mathrm{S}$ phase (Fig. 1) [8, 9]. The monoubiquitinated ID complex forms foci on damaged DNA. FANCM is also a crucial gene, as it is a sensor for detecting stalled DNA replication. The BRCA complex, which is also referred to as homologous recombination (HR), consists of FANCD1, FANCJ, FANCN, and FANCO, and is located downstream of the DI complex on the ICL repair pathway.
Table 1 Genes mutated in patients with Fanconi anemia

\begin{tabular}{llll}
\hline & $\begin{array}{l}\text { Other } \\
\text { names }\end{array}$ & $\begin{array}{l}\text { Chromosomal } \\
\text { Locus }\end{array}$ & $\begin{array}{l}\text { Population in FA } \\
\text { patients }(\%)\end{array}$ \\
\hline FANCA & & $16 \mathrm{q} 24.3$ & $60-70$ \\
FANCB & & Xp22.2 & 2 \\
FANCC & & $9 \mathrm{q} 22.3$ & 14 \\
FANCD1 & BRCA2 & $13 \mathrm{q} 13.1$ & 3 \\
FANCD2 & & $3 \mathrm{p} 25.3$ & 3 \\
FANCE & & $6 \mathrm{p} 22-\mathrm{p} 21$ & 3 \\
FANCF & & $11 \mathrm{p} 15$ & 2 \\
FANCG & & $9 \mathrm{p} 13$ & 10 \\
FANCI & & $15 \mathrm{q} 25-\mathrm{q} 26$ & 1 \\
FANCJ & BACH1/BRIP1 & $17 \mathrm{q} 22$ & 2 \\
FANCL & & $2 \mathrm{p} 16.1$ & 0.20 \\
FANCM & & $14 \mathrm{q} 21.3$ & 0.20 \\
FANCN & PALB2 & $16 \mathrm{p} 12.3-\mathrm{p} 12.2$ & 0.70 \\
FANCO & RAD51C & $17 \mathrm{q} 22$ & 0.20 \\
FANCP & SLX4 & $16 \mathrm{p} 13.3$ & 0.20 \\
\hline
\end{tabular}

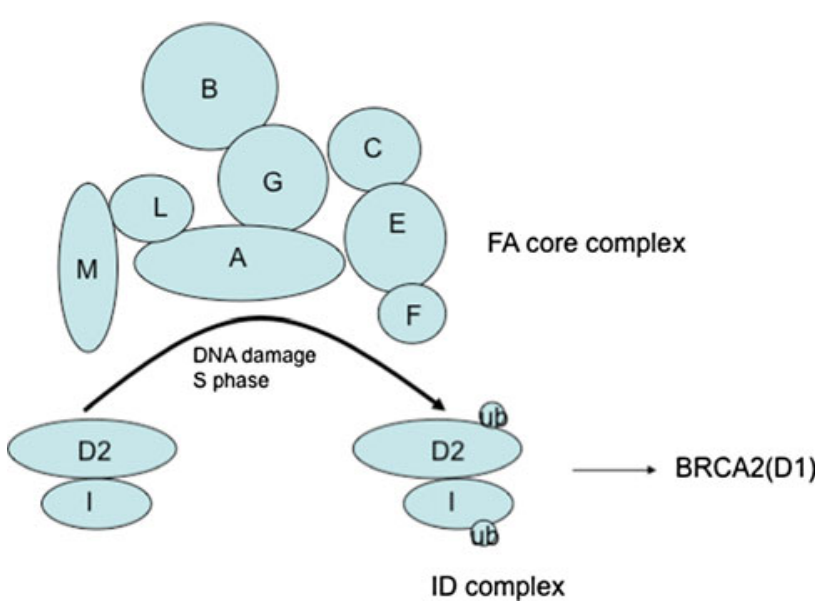

Fig. 1 Simplified scheme of the FA pathway. Depending on the FA core complex, FANCD2 and FANCI are monoubiquitinated after DNA damage or during the S-phase

Prognosis factors in FA patients

Although FANC gene knockout mice models have been established, they differ from the hematological phenotypes of human FA patients [10]. With the exception of the lethal phenotype of the BRCA2/FANCD1 knockout mouse, the hematological parameters of the other FA groups show only a slightly decreased platelet count and a slightly increased erythrocyte mean cell volume in mice at a young age, which did not progress to aplastic anemia or leukemia. However, both male and female mice showed hypogonadism and impaired fertility, which is consistent with the human FA patient phenotypes. 
Recent studies have revealed a relationship between the acetaldehyde and FA pathways. Acetaldehyde is an organic chemical compound that is naturally present in coffee, bread, and ripe fruit, which is produced as product of a plant's normal metabolism. It is also produced by the oxidation of ethylene. In the liver, the enzyme alcohol dehydrogenase $(\mathrm{ADH})$ oxidizes ethanol into acetaldehyde, which is then further oxidized into harmless acetic acid by acetaldehyde dehydrogenase (ALDH). These oxidation reactions are coupled with the reduction of $\mathrm{NAD}^{+}$to NADH. ALDH2, an isozyme of ALDH, contains the functional polymorphism, ALDH2 Glu487Lys. An association between this polymorphism and squamous cell carcinomas, such as esophageal cancer in alcoholics, has been reported. A recent study reported that exposure of cells to acetaldehyde results in a concentration-dependent increase in FANCD2 monoubiquitination [11]. Acetaldehyde also stimulates BRCA1 phosphorylation at Ser1524 and increases the level of H2AX, a marker of homologous recombination. Both modifications occur in a dose-dependent manner.

Another report showed that ALDH2 is essential for the development of FANCD 2(-/-) embryos [12]. Nevertheless, mothers with AA enzyme (ALDH $2(+/-)$ ) can support the development of double-mutant (ALDH2 $(-/-)$ FANCD2(-/-)) mice. These embryos are unusually sensitive to ethanol exposure in utero, with ethanol consumption by postnatal double-deficient mice rapidly precipitating bone marrow failure. ALDH2 $(-/-)$ FANCD2(-/-) mice also spontaneously develop acute leukemia.

This previous study also provided the first evidence of the factors responsible for driving the FA hematological phenotype in mice. DNA damage caused by acetaldehyde may contribute critically to the genesis of fetal alcohol syndrome in fetuses, as well as to abnormal development, bone marrow failure, and cancer predisposition in FA patients. This research group also focused on hematopoietic stem cells (HSCs) in another study [13]. They reported finding that some aged ALDH2(-/-)FANCD2(-/-) mutant mice that did not develop leukemia spontaneously developed aplastic anemia, with a concomitant accumulation of damaged DNA within the hematopoietic stem and progenitor cell (HSPC) pool. Only HSPCs and not the more mature blood precursors require Aldh2 for protection against acetaldehyde toxicity. There is more than a 600-fold reduction in the HSC pool of mice deficient in both FA pathway-mediated DNA repair and acetaldehyde detoxification. This study data indicated that the emergence of bone marrow failure in FA was probably due to aldehyde-mediated genotoxicity restricted to the HSPC pool.

All of the ALDH data suggest that ALDH2 polymorphism is critical to the prognosis of FA patients.
Intercrosslink repair

DNA ICLs are toxic to dividing cells, as they induce mutations, chromosomal rearrangements, and cell death. In order to survive, organisms have developed strategies for dealing with DNA damage. As such, specialized repair pathways have evolved for specific kinds of DNA damage, including double-strand break (DSB) and ICL. Inducers of ICLs are important drugs in cancer treatment and include the well-known chemotherapeutic agents mitomycin C, cisplatin, cyclophosphamide, and their respective derivatives. While cells derived from most individuals with FA are hypersensitive to ICLs, they are generally not hypersensitive to inducers of DSBs such as ionizing radiation, indicating that the ICL repair pathway is distinct from that of DSB.

Homologous recombination is a DNA repair pathway that utilizes strand exchange in a gene conversion reaction involving a single-strand and a DNA duplex. In mammalian cells, this is a major repair pathway for DNA damage such as DSBs. The strand exchange protein RAD51 and the products of the hereditary breast cancer susceptibility genes BRCA1 and BRCA2 [14, 15] are critical proteins in HR in mammalian cells.

In 2005, a cellular study in humans showed that mutation of either the FA core complex members or the FANCD2 monoubiquitination site resulted in HR defects [16]. These defects, however, are mild compared with those resulting from a BRCA2 deficiency. HR measurements in these previous studies were performed with the widely used DR-GFP reporter system, in which a DSB formed by I-SceI endonuclease results in green fluorescent protein-expressing (GFP+) cells repaired by HR (Fig. 2). Further studies have reported on the mechanisms of ICL repair, particularly in terms of the replication-coupled manner. A 2008 study using a cell-free system based on Xenopus egg extracts found that ICL is repaired in a replication-dependent manner [17]. Another study in the Xenopus egg showed that ubiquitinated FANCI-FANCD2 is essential for replication-dependent ICL repair and that it is able to control the incision step [18]. Development and use of a TR-GFP assay, a modified version of the DR-GFP HR assay system, demonstrated that ICL repair in mammalian cells is dependent on DNA replication. The TRGFP assay uses a DNA template with a site-specific ICL at sequences that are complemented to triplex-forming oligonucleotide conjugated with psoralen (pso-TFO) [19]. The construct also contains an origin of replication from the Epstein-Barr virus (EBV), enabling replication in human cells. Their results showed that ICL-induced HR was substantially compromised in the absence of FA proteins, suggesting that the FA pathway is specifically involved in replication-coupled HR repair. Use of direct assays for ICL-induced HR in vivo, along with studies that 


\section{DR/TR-GFP}

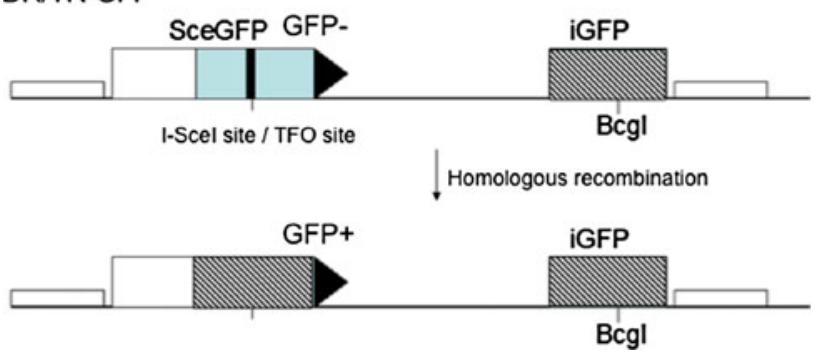

Fig. 2 DR-GFP assay. In the $D R-G F P$ substrate, an I-SceI site is inserted into the GFP gene on sceGFP. GFP is inactivated by the stopcodon in the I-SceI site. To restore functionality of the GFP gene, the $i G F P$ gene has $.8 \mathrm{~kb}$ of sequence homology to direct the repair of an I-SceI-cleaved SceGFP gene

have demonstrated the involvement of the FA pathway overall, may facilitate delineation of the mechanisms and factors involved in this process.

\section{Diamond-Blackfan anemia}

Diamond-Blackfan anemia (DBA) is a rare congenital bone marrow failure syndrome characterized by severe normochromic macrocytic anemia and reticulocytopenia, with selective hypoplasia of erythroid precursors in the bone marrow. Up to $50 \%$ of affected individuals have physical abnormalities including short stature, craniofacial dysmorphism, heart defects, and anomalies of the thumbs and genitourinary tract [20]. Increased risk of malignant disease, such as acute myeloid leukemia and osteogenic sarcoma, has also been reported to occur in this syndrome [21]. The incidence of DBA has been estimated to be 5-7 per million live births in Europe and North America. In a national study conducted in Japan between 2006 and 2010, 65 new DBA patients were registered. During this study period, the mean number of live births per year in Japan was reported to be 1.08 million, putting the incidence of DBA at 12 per each million live births, as most of these patients were diagnosed as DBA during infancy. The majority of these patients are sporadic, with the percentages of patients with autosomal dominant inheritance reported to be less than $10 \%$. Corticosteroids are recommended as a first line therapy, as these have been reported to improve erythropoiesis in approximately $80 \%$ of DBA patients. In patients refractory to corticosteroids or who develop other forms of cytopenia, HSC transplantation has been suggested as a viable alternative [22].

Molecular pathogenesis

The first DBA gene (RPS19) was identified in 1999 and was found in approximately $25 \%$ of the probands in western countries [23]. Since then, a total of nine genes encoding large (RPL) or small (RPS) ribosomal subunit proteins were found to be mutated in DBA patients, including RPL5 (6.6 \%), RPL11 (4.8 \%), RPL35A (3\%), RPS24 (2\%), RPS17 (1\%), RPS7 (1\%), RPS10 (6.4\%), and RPS26 (2.6\%) [24] (Table 2). Collectively, mutations in at least one of these nine genes have been detected in approximately $50-60 \%$ of DBA patients. Of 68 Japanese been examined, mutations in RPS 19, RPL5, RPL11, RPS17, RPS26 were identified in $10(14.7 \%)$, six (8.8\%), three $(4.4 \%)$, one $(1.5 \%)$, one $(1.5 \%)$, and one $(1.5 \%)$, respectively. These mutations have subsequently been determined to occur in $32.4 \%$ of Japanese patients [25, 26]. A low incidence of mutations in the RPS19 gene may account for the overall lower incidence of total mutations in the Japanese population.

As conventional gene sequencing cannot identify large gene deletions, there have been only a few reports of patients with allelic losses in the RPS19 and RPL35A genes. Kuramitsu et al. [26] investigated large deletions of the RP genes using gene copy number variation analysis based on a quantitative-PCR and a single-nucleotide polymorphism (SNP) array. This study used sequencing to screen for large gene deletion in 27 patients without gene mutations. The PCR-based gene copy number assay identified a large deletion in seven $(25.9 \%)$ of 27 patients. Of these, three patients had RPS17, two had RPS19, one had RPL5, while one had RPL35A deletions. The SNP array confirmed six of the seven large deletions. Based on these new methods, the frequency of RP gene abnormalities in the DBA patients increased to $42.6 \%$. All patients with large deletions in DBA genes exhibited malformation with growth retardation. However, half of the patients with a mutation due to sequencing had growth retardation, while all seven patients with a large deletion exhibited growth retardation. While four of seven patients responded to corticosteroids, there were no phenotypic

Table 2 Ribosomal protein gene mutations and deletions in 68 Japanese patients with Diamond-Blackfan anemia

\begin{tabular}{llll}
\hline Gene & Mutation & Deletion & Total $(\%)$ \\
\hline RPS 19 & 10 & 2 & $12(17.6 \%)$ \\
RPL 5 & 6 & 1 & $7(10.3 \%)$ \\
RPS 17 & 3 & 0 & $3(4.4 \%)$ \\
RPL 11 & 3 & 1 & $4(5.9 \%)$ \\
RPS 10 & 1 & 0 & $1(1.5 \%)$ \\
RPS 26 & 1 & 0 & $1(1.5 \%)$ \\
RPS 35A & 0 & 1 & $1(1.5 \%)$ \\
RPS 24 & 0 & 0 & 0 \\
RPS 14 & 0 & 0 & 0 \\
Total & 22 & 7 & $29(42.6 \%)$ \\
\hline
\end{tabular}


differences noted between patients with and without large deletions, including response rate to corticosteroids and other malformations.

Farrar et al. [27] also identified RP gene deletions in nine $(17 \%)$ of 51 patients without any identifiable mutation by SNP array. Of these nine patients, three had RPS17, two had RPS26, two had RPS19, and two had RPL35A deletions. Clinically, five of the nine patients responded to corticosteroids. Two exhibited short stature. These two studies suggested that genomic deletions may be detected in $4-10 \%$ of DBA patients, which is more common than has been previously suspected. Thus, in addition to conventional gene sequencing, molecular studies of suspected DBA cases should also include either a SNP array or PCRbased gene copy number assay.

Despite extensive sequencing of all the RP genes, at present mutations have only been found in approximately half of DBA patients examined, which raises the question whether other genes are responsible for DBA. Recent advance in genomic sequencing have made it possible to search for new candidate genes. Sankaran et al. [28] performed exome sequencing on two siblings without RP gene mutations. Both affected siblings satisfied the diagnostic criteria for DBA and both parents had normal blood values, suggesting X-linked or autosomal recessive inheritance. During sequencing, at least 10 -fold coverage was obtained in more than $93 \%$ of the target bases. After filtering, a total of 74 variants were identified as being shared by the three affected siblings. Of these 74 mutations, 31 were found in two affected siblings but not in the unaffected sibling. No variants were identified that would fit an autosomal recessive model of inheritance. Only the GATA1 gene showed appropriate segregation for an X-linked disease with full penetrance. The mutation in the GATA1 gene is a G-C transversion at position $48,649,736$ on the X chromosome and results in a substitution of leucine for valine at amino acid 74 of the GATA1 protein. This mutation impaired production of the full-length form of the exon 2 protein. After screening 62 additional male DBA patients without known mutations for the GATA1 mutation, the study also identified one patient with a mutation in GATA1 at the exon 2-intron 2 junction. It was predicted that this would result in impaired splicing and a frameshift of the full-length GATA1 open reading frame. Overall, this study has opened new avenues for studying the molecular pathogenesis of DBA.

Role of p53 in the pathophysiology of DBA

Although current evidence suggests that impaired ribosomal biogenesis should affect all blood cell lineages, one question remains as to why it affects only the erythroid progenitors. Several animal models have demonstrated the role of p53 in the pathophysiology of DBA. The RPS19deficient zebrafish model has been shown to have many features of DBA and is accompanied by the up-regulation of the p53 family [29]. Suppression of p53 in the RPS19deficient zebrafish alleviated the phenotype and improved survival. RPS19 knockdown mouse fetal liver cells, which were created by retrovirus-infected siRNA, showed reduced proliferation but normal differentiation of erythroid cells and an increased level of p53 and p21 [30]. Dutt et al. [31] have examined the accumulation and activity of p53 in different hematopoietic lineages after a partial knockdown of the RPS19 gene in primary human bone marrow-derived CD34 cells. Their study showed that p53 accumulates selectively in erythroid progenitors, resulting in lineage-specific p53 target gene expression, cell cycle arrest, and apoptosis. While pifithrin- $\alpha$ has been shown to inhibit the activity of p53, nutlin-3 activates p53 through the inhibition of HDM2. In addition, nutlin-3 selectivity impairs erythropoiesis, whereas inhibition of p53 by pifithrin- $\alpha$ rescues the erythroid defect. To directly examine whether p53 accumulation is operative in patients with DBA, bone marrow biopsies from eight patients with DBA were stained with anti-human p53 antibody and shown to have strong nuclear staining in two patients and weak nuclear staining in six patients. The erythroid lineage has a low threshold for the induction of p53, which accounts for the selective impaired erythropoiesis in patients with DBA.

\section{Alternative therapies for DBA}

Although approximately $80 \%$ of DBA patients initially respond to corticosteroid, half of the responders are steroiddependent. Only $20 \%$ of these patients achieve remission. Although historically many alternative drugs have been tried, there has been no agreement on a second-line therapy. L-leucine is an essential amino acid and is known to be an activator of mRNA and stimulate protein synthesis through the mammalian target of rapamycin (mTOR) pathway. L-leucine treatment of the RPS19-deficient zebrafish model results in a striking improvement of anemia and developmental defects. These findings were reproduced in primary human CD34 cells after knockdown of the RPS19 gene [32]. Therapeutic effect of L-leucine has also been confirmed in the mouse model for RPS19-deficient DBA and shown to be associated with reduced p53 activity in hematopoietic progenitors [33]. Recently, leucine has been used on an investigational basis in one patient with DBA and is reported to have achieved a remission [34].

These findings support commencement of a clinical trial with L-leucine as an alternative therapy for DBA. 


\section{Dyskeratosis congenita}

Clinical features of patients with dyskeratosis congenita

Dyskeratosis congenita (DC) is a rare inherited disease characterized by the classical mucocutaneous triad of abnormal skin pigmentation, nail dystrophy, and mucosal leucoplakia in approximately $80-90 \%$ of patients [34]. Patients with DC are unable to maintain the telomere complex that protects the chromosome ends and consequently have very short telomeres [35]. Shortened telomeres can cause a wide variety of clinical features across a phenotypic spectrum consisting not only of mucocutaneous abnormalities but also multisystem symptoms including bone marrow failure, pulmonary fibrosis, hepatic fibrosis, and predisposition to malignancy [36, 37]. Indeed, nonmucocutaneous features, such as bone marrow failure and pulmonary fibrosis, occasionally precede mucocutaneous abnormalities, making it difficult to diagnose patients with DC based on clinical features alone. The incidence of DC is estimated to be one per million live births.

The diagnostic criteria for DC proposed by Vulliamy [38] include one or more of the three classic mucocutaneous features combined with hypoplastic bone marrow and at least two other somatic features known to occur in DC. The primary causes of mortality in patients with DC are bone marrow failure syndrome (60-70 \%), pulmonary complications (10-15\%), and malignancy (particularly MDS and AML) $(10 \%)[36,37]$.

\section{Genetic background of DC}

DC is a genetically heterogeneous disorder, showing autosomal recessive, autosomal dominant, and X-linked inheritance. The DKC1 gene on chromosome (chr) Xq28, which encodes dyskerin, was the first gene identified in the $\mathrm{X}$-linked DC patients [39]. Dyskerin has a close association with the RNA component of telomerase (TERC), and mutations in dyskerin cause a reduction in accumulation of $T E R C$ and reduced telomere length [35]. In addition to its role in the biogenesis of telomerase RNA dyskerin is involved in ribosomal RNA biogenesis. Dyskerin catalyzes uridine to pseudouridine, which is a critical step for ribosomal RNA maturation and function. These findings imply that both telomere and ribosomal defects may occur in patients with $D K C 1$ mutations. Subsequently, heterozygous TERC mutations have also been found in autosomal dominant DC patients [40]. Genetic screening has identified mutations of other components of the telomerase complex, including TERT (chr 5p15) [41, 42], NOP10 (chr 15q14q15) [43], and NHP2 (chr 5q35) [44] in patients with rare autosomal recessive DC. Mutations of TERT have also been reported in the autosomal dominant family [45].
Moreover, heterozygous mutations of TINF2 (chr 14q12) that encode TIN2, which is the main component of shelterin and which protects telomeres, have been identified in $<11 \%$ of DC patients [46, 47].

More recently, mutations of TCAB1 (chr 17p13) were identified in patients with $\mathrm{DC}$ as autosomal recessive forms [48]. Venteicher et al. [49] found that TCAB1 associates with TERT, dyskerin and TERC, and small Cajal body RNAs (scaRNAs) that are involved in modifying splicing RNAs to control telomerase trafficking. TCAB1 defects prevented TERC from associating with the Cajal bodies, which disrupted the telomerase-telomere association. A recent case report described biallelic mutations of the CTCl gene (chr 17p13) in a patient with DC [50]. This gene was originally described as causative gene of the Coats plus syndrome, which is a form of cerebroretinal microangiopathy with calcifications and cysts (CRMCC). The mutation frequencies of these new genes for DC remain unknown.

At present, eight of the mutated genes in DC have been shown to be associated with the telomerase holoenzyme (TERT, TERC, DKC1, NOP10, NHP2, TCAB1, and CTC1) or the shelterin complex (TINF2), accounting for approximately $50 \%$ of DC patients. Mutations in telomerase and telomere components have also been identified in patients with aplastic anemia, pulmonary fibrosis, and liver diseases that did not have any mucocutaneous manifestations [45, 46, 51-59]. These findings suggest that defective telomere maintenance causes not only classical DC, but also a broad spectrum of diseases previously thought to be idiopathic and thus this has led to a new concept of diseases termed "syndromes of telomere shortening".

\section{Cryptic DC patients in aplastic anemia}

Patients with DC have been shown to have disease diversity in terms of age at onset, symptoms, and severity. This diversity occurs even among the patients with the same gene mutation. Bone marrow failure sometimes precedes mucocutaneous manifestations in patients with DC, and a substantial proportion of patients with aplastic anemia have shorter telomeres compared with normal individuals [60, 61]. These observations have prompted screening for gene mutations responsible for telomere maintenance in patients with aplastic anemia and other bone marrow failure syndromes. This screening identified mutations in TERC and TERT in $3 \%$ of the aplastic anemia patients [54, 55]. Our research group conducted a study in Japanese children with aplastic anemia and identified two of 96 as having the TERT mutations, although none of the patients had a TERC mutation [53]. Patients with TERC or TERT mutations have been shown to have very short telomeres in their blood cells. Recently, Du et al. [52] found that 6 (5.5\%) of 109 
pediatric patients with severe aplastic anemia had mutations of TINF2. In an unpublished study, our research group screened for mutations of TINF2 and found that of the 96 pediatric patients with aplastic anemia that were examined, none exhibited any mutations of this gene.

Three methods are commonly used for measuring telomere length, including Southern blot, real-time polymerase chain reaction, and flow cytometry and fluorescence in situ hybridization (flow-FISH). Of these, the flow-FISH has been shown to be the most appropriate when undertaking "prospective" screening [62, 63]. As shown in Fig. 3, patients with DC and aplastic anemia with the TERT mutation were all found to have very short telomeres as compared with the idiopathic aplastic anemia patients and normal individuals. As a small subset of patients with apparently idiopathic aplastic anemia have been shown to carry telomere gene mutations, identification of such patients is critical for informing treatment decisions. Aplastic anemia patients should be routinely screened for telomere gene mutations prior to starting any treatment. However, because screening of gene mutations can be both laborious and time consuming, we have adopted the screening of telomere length in blood cells rather than screening of gene mutations.

It should be noted that short telomeres are not specific for patients with DC, as they are also seen in patients with other bone marrow failure syndromes. Although short telomeres have also been found in patients with other congenital bone marrow failure syndromes, such as Shwachman-Diamond syndrome and Fanconi anemia, telomere lengths in patients with DC have been demonstrated to be

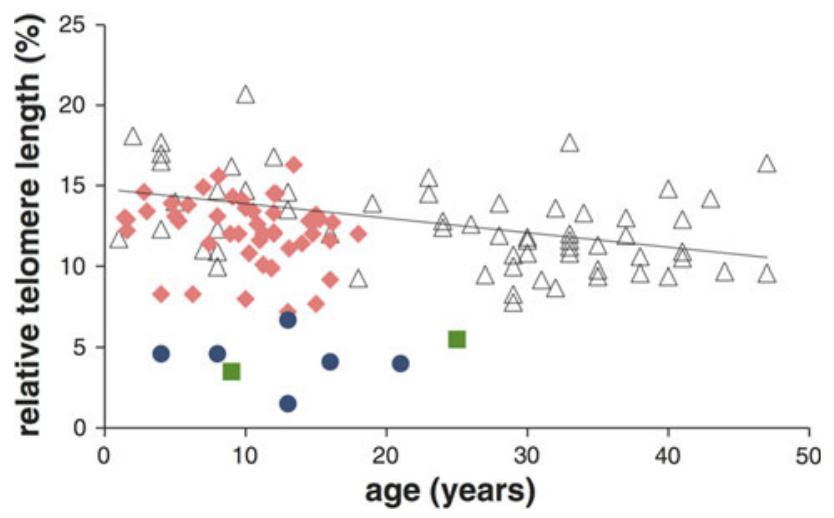

Fig. 3 Relative telomere length in peripheral blood lymphocytes from patients with dyskeratosis congenita (filled circles), patients with aplastic anemia harboring TERT mutations (filled squares), patients with idiopathic aplastic anemia (filled argyles) and normal individuals (open triangles). Telomere lengths were measured by flow cytometryfluorescent in situ hybridization (flow-FISH). Relative telomere length was calculated as the ratio between the telomere signal of each sample and the telomere signal of the control cell line (cell line 1301). These data were provided by the Department of Pediatrics, Nagoya University Graduate School of Medicine shorter than those in all other bone marrow failure syndromes. In fact, telomere length in most patients with DC is below the first percentile of telomere length found in healthy controls [64].

Family members of patients with DC should receive genetic counseling to rule out if they are silent carriers. In particular, genetic counseling is necessary during the proband search for a donor for HSC transplantation. Studies on telomere length analyses in families with DC have shown that mutated carriers with clinical signs of bone marrow failure have short telomeres. Even so, telomere length cannot predict the presence or absence of a mutation in family members with bone marrow failure. In addition, there have been rare cases that show normal telomere length, even though the subject harbors the same mutation as the proband. This suggests that mutation alone does not sufficiently explain the reduction of telomere length [51].

\section{Clinical management for DC}

Bone marrow failure and immune deficiency are the most common causes of death in up to $60-70 \%$ of patients with DC. Androgen (e.g. oxymetholone) has been used to improve cytopenia in patients with DC since the 1960s. However, the mechanism of action of androgen has not been well understood until recently. Calado et al. [65] showed that in vitro exposure of normal peripheral blood cells to androgen produced higher TERT mRNA levels. When these patients were treated with cells from patients who had a heterozygous mutation of the telomerase, it was possible to restore their low baseline telomerase activity to normal levels. Thus, as telomere shortening is closely associated with malignant disease, androgen therapy might be able to prevent or postpone the development of various types of cancers. Erythropoietin and/or G-CSF combined with androgen has occasionally provided transient hematopoietic recovery to poor responders to androgen alone [66]. However, this combination should be used with caution, as severe splenic peliosis and fatal rupture have been reported in two patients with DC who received simultaneous administration of androgen and G-CSF [67].

Allogeneic HSC transplantation is the only curative treatment for bone marrow failure in patients with DC. However, the outcome in previous reports has been disappointing due to unacceptable transplant-related toxicities, including severe pulmonary/liver complications, especially in transplants from an alternative donor $[68,69]$. To avoid these complications, non-myeloablative conditioning regimens have been recently used in several cases. Dietz et al. [70] reported encouraging results of six patients with DC who received a fludarabine-based nonmyeloablative regimen. Of the four surviving patients, three were recipients of unrelated grafts. Non-myeloablative 
transplants are expected to provide improvement in the short-term survival. At our institute, three patients with DC underwent allogeneic bone marrow transplantation following non-myeloablative conditioning from 2003 to 2009. Successful engraftment was achieved in all patients with only a few regimen-related toxicities, and at the present time all continue to survive without any symptoms [71]. However, due the late effects of conditioning agents and allogeneic immune responses within the recipient's organs, such as the lung and liver, longer-term follow-ups are necessary to definitively clarify the present results.

\section{Conclusion}

Although recent studies have identified many causative genes, mutations of these genes have only been found in half of the patients with DBA or DKC. Next-generation sequencing (or massive parallel sequencing) technologies have led to a tremendous revolution in genomics, with their effects currently becoming increasingly widespread. This new strategy may soon be able to reveal the remaining unknown causative genes in IBMFS.

Recently, Agarwal et al. [72] established induced pluripotent stem cells (iPSCs) derived from a patient with DC and showed that the reprogrammed DC cells overcame a critical limitation in TERC levels to restore the telomere maintenance and self-renewal. These findings indicate that drugs or gene therapy that upregulate TERC activity may show therapeutic potential in patients with DC. These same strategies may also be applicable for other IBMFS.

The only long-term curative treatment for bone marrow failure in patients with IBMFS is allogeneic HSC transplantation, although this procedure has a risk of severe adverse effects. Multicenter prospective studies are needed to establish appropriate conditioning regimens aimed at reducing transplant-related mortality. Future studies must aim to improve short-term outcomes, such as hematological recovery, and to decrease the incidence of late adverse effects.

\section{References}

1. Joenje H, Patel KJ. The emerging genetic and molecular basis of Fanconi anaemia. Nat Rev Genet. 2001;2:446-57.

2. Kim H, D'Andrea AD. Regulation of DNA cross-link repair by the Fanconi anemia/BRCA pathway. Genes Dev. 2012;26: 1393-408.

3. D'Andrea AD, Grompe M. The Fanconi anaemia/BRCA pathway. Nat Rev Cancer. 2003;3:23-34.

4. Alter BP, Rosenberg PS, Brody LC. Clinical and molecular features associated with biallelic mutations in FANCD1/BRCA2. J Med Genet. 2007;44:1-9.
5. Rosenberg PS, Socie G, Alter BP, Gluckman E. Risk of head and neck squamous cell cancer and death in patients with Fanconi anemia who did and did not receive transplants. Blood. 2005; 105:67-73.

6. Whitney MA, Saito H, Jakobs PM, Gibson RA, Moses RE, Grompe M. A common mutation in the FACC gene causes Fanconi anaemia in Ashkenazi Jews. Nat Genet. 1993;4:202-5.

7. Gibson RA, Hajianpour A, Murer-Orlando M, Buchwald M, Mathew CG. A nonsense mutation and exon skipping in the Fanconi anaemia group C gene. Hum Mol Genet. 1993;2:797-9.

8. Garcia-Higuera I, Taniguchi T, Ganesan S, Meyn MS, Timmers $\mathrm{C}$, Hejna $\mathrm{J}$, et al. Interaction of the Fanconi anemia proteins and BRCA1 in a common pathway. Mol Cell. 2001;7:249-62.

9. Smogorzewska A, Matsuoka S, Vinciguerra P, McDonald ER 3rd, Hurov KE, Luo J, et al. Identification of the FANCI protein, a monoubiquitinated FANCD2 paralog required for DNA repair. Cell. 2007;129:289-301.

10. Carreau M. Not-so-novel phenotypes in the Fanconi anemia group D2 mouse model. Blood. 2004;103:2430.

11. Marietta C, Thompson LH, Lamerdin JE, Brooks PJ. Acetaldehyde stimulates FANCD2 monoubiquitination, H2AX phosphorylation, and BRCA1 phosphorylation in human cells in vitro: implications for alcohol-related carcinogenesis. Mutat Res. 2009;664:77-83.

12. Langevin F, Crossan GP, Rosado IV, Arends MJ, Patel KJ. Fancd 2 counteracts the toxic effects of naturally produced aldehydes in mice. Nature. 2011;475:53-8.

13. Garaycoechea JI, Crossan GP, Langevin F, Daly M, Arends MJ, Patel KJ. Genotoxic consequences of endogenous aldehydes on mouse haematopoietic stem cell function. Nature. 2012;489: $571-5$.

14. Moynahan ME, Chiu JW, Koller BH, Jasin M. Brca1 controls homology-directed DNA repair. Mol Cell. 1999;4:511-8.

15. Moynahan ME, Pierce AJ, Jasin M. BRCA2 is required for homology-directed repair of chromosomal breaks. Mol Cell. 2001;7:263-72.

16. Nakanishi K, Yang YG, Pierce AJ, Taniguchi T, Digweed M, D'Andrea AD, et al. Human Fanconi anemia monoubiquitination pathway promotes homologous DNA repair. Proc Natl Acad Sci USA. 2005;102:1110-5.

17. Raschle M, Knipscheer P, Enoiu M, Angelov T, Sun J, Griffith JD, et al. Mechanism of replication-coupled DNA interstrand crosslink repair. Cell. 2008;134:969-80.

18. Knipscheer P, Raschle M, Smogorzewska A, Enoiu M, Ho TV, Scharer OD, et al. The Fanconi anemia pathway promotes replication-dependent DNA interstrand cross-link repair. Science. 2009;2009(326):1698-701.

19. Nakanishi K, Cavallo F, Perrouault L, Giovannangeli C, Moynahan ME, Barchi M, et al. Homology-directed Fanconi anemia pathway cross-link repair is dependent on DNA replication. Nat Struct Mol Biol. 2011;18:500-3.

20. Alter BP, Young NS. The bone marrow failure syndromes. In: Nathan DG, Orkin HS, editors. Hematology of infancy and childhood, vol 1. Philadelphia: Saunders; 1998. p. 237-335.

21. Vlachos A, Rosenberg PS, Atsidaftos E, Alter BP, Lipton JM. Incidence of neoplasia in Diamond Blackfan anemia: a report from the Diamond Blackfan Anemia Registry. Blood. 2012;119: 3815-9.

22. Mugishima H, Ohga S, Ohara A, Kojima S, Fujisawa K, For the Aplastic Anemia Committee of the Japanese Society of Pediatric Hematology. Hematopoietic stem cell transplantation for Diamond-Blackfan anemia: a report from the Aplastic Anemia Committee of the Japanese Society of Pediatric Hematology. Pediatr Transpl. 2007;11:601-7.

23. Draptchinskaia N, Gustavsson P, Andersson B, Pettersson M, Willig TN, Dianzani I, et al. The gene encoding ribosomal 
protein $\mathrm{S} 19$ is mutated in Diamond-Blackfan anaemia. Nat Genet. 1999;21:169-75.

24. Boria I, Garelli E, Gazda HT, Aspesi A, Quarello P, Pavesi E, et al. The ribosomal basis of Diamond-Blackfan anemia: mutation and database update. Hum Mutat. 2010;31:1269-79.

25. Konno Y, Toki T, Tandai S, Xu G, Wang R, Terui K, et al. Mutations in the ribosomal protein genes in Japanese patients with Diamond-Blackfan anemia. Haematologica. 2010;95: 1293-9.

26. Kuramitsu M, Sato-Otsubo A, Morio T, Takagi M, Toki T, Terui $\mathrm{K}$, et al. Extensive gene deletions in Japanese patients with Diamond-Blackfan anemia. Blood. 2012;119:2376-84.

27. Farrar JE, Vlachos A, Atsidaftos E, Carlson-Donohoe H, Markello TC, Arceci RJ, et al. Ribosomal protein gene deletions in Diamond-Blackfan anemia. Blood. 2011;118:6943-51.

28. Sankaran VG, Ghazvinian R, Do R, Thiru P, Vergilio JA, Beggs $\mathrm{AH}$, et al. Exome sequencing identifies GATA1 mutations resulting in Diamond-Blackfan anemia. J Clin Invest. 2012;122: 2439-43.

29. Danilova N, Sakamoto KM, Lin S. Ribosomal protein S19 deficiency in zebrafish leads to developmental abnormalities and defective erythropoiesis through activation of p53 protein family. Blood. 2008;112:5228-37.

30. Sieff CA, Yang J, Merida-Long LB, Lodish HF. Pathogenesis of the erythroid failure in Diamond Blackfan anaemia. Br J Haematol. 2010;148:611-22.

31. Dutt S, Narla A, Lin K, Mullally A, Abayasekara N, Megerdichian $\mathrm{C}$, et al. Haploinsufficiency for ribosomal protein genes causes selective activation of p53 in human erythroid progenitor cells. Blood. 2011;117:2567-76.

32. Payne EM, Virgilio M, Narla A, Sun H, Levine M, Paw BH, et al. L-leucine improves the anemia and developmental defects associated with Diamond-Blackfan anemia and del(5q) MDS by activating the mTOR pathway. Blood. 2012;120:2214-24.

33. Jaako P, Debnath S, Olsson K, Bryder D, Flygare J, Karlsson S. Dietary L-leucine improves the anemia in a mouse model for Diamond-Blackfan anemia. Blood. 2012;120:2225-8.

34. Kirwan M, Dokal I. Dyskeratosis congenita, stem cells and telomeres. Biochim Biophys Acta. 2009;1792:371-9.

35. Mitchell JR, Wood E, Collins K. A telomerase component is defective in the human disease dyskeratosis congenita. Nature. 1999;402:551-5.

36. Dokal I. Dyskeratosis congenita in all its forms. Br J Haematol. 2000;110:768-79.

37. Walne AJ, Dokal I. Advances in the understanding of dyskeratosis congenita. Br J Haematol. 2009;145:164-72.

38. Vulliamy TJ, Marrone A, Knight SW, Walne A, Mason PJ, Dokal I. Mutations in dyskeratosis congenita: their impact on telomere length and the diversity of clinical presentation. Blood. 2006;107: 2680-5.

39. Heiss NS, Knight SW, Vulliamy TJ, Klauck SM, Wiemann S, Mason PJ, Poustka A, Dokal I. X-linked dyskeratosis congenita is caused by mutations in a highly conserved gene with putative nucleolar functions. Nat Genet. 1998;19:32-8.

40. Vulliamy T, Marrone A, Goldman F, Dearlove A, Bessler M, Mason PJ, et al. The RNA component of telomerase is mutated in autosomal dominant dyskeratosis congenita. Nature. 2001;413: 432-5.

41. Armanios M, Chen JL, Chang YP, Brodsky RA, Hawkins A, Griffin CA, et al. Haploinsufficiency of telomerase reverse transcriptase leads to anticipation in autosomal dominant dyskeratosis congenita. Proc Natl Acad Sci USA. 2005;102:15960-4.

42. Marrone A, Walne A, Tamary H, Masunari Y, Kirwan M, Beswick $\mathrm{R}$, et al. Telomerase reverse-transcriptase homozygous mutations in autosomal recessive dyskeratosis congenita and Hoyeraal-Hreidarsson syndrome. Blood. 2007;110:4198-205.
43. Walne AJ, Vulliamy T, Marrone A, Beswick R, Kirwan M, Masunari Y, et al. Genetic heterogeneity in autosomal recessive dyskeratosis congenita with one subtype due to mutations in the telomerase-associated protein NOP10. Hum Mol Genet. 2007;16: 1619-29.

44. Vulliamy T, Beswick R, Kirwan M, Marrone A, Digweed M, Walne A, et al. Mutations in the telomerase component NHP2 cause the premature ageing syndrome dyskeratosis congenita. Proc Natl Acad Sci USA. 2008;105:8073-8.

45. Vulliamy TJ, Walne A, Baskaradas A, Mason PJ, Marrone A, Dokal I. Mutations in the reverse transcriptase component of telomerase (TERT) in patients with bone marrow failure. Blood Cells Mol Dis. 2005;34:257-63.

46. Walne AJ, Dokal I. Dyskeratosis congenita: a historical perspective. Mech Ageing Dev. 2008;129:48-59.

47. Savage SA, Giri N, Baerlocher GM, Orr N, Lansdorp PM, Alter BP. TINF2, a component of the shelterin telomere protection complex, is mutated in dyskeratosis congenita. Am J Hum Genet. 2008;82:501-9.

48. Zhong F, Savage SA, Shkreli M, et al. Disruption of telomerase trafficking by TCAB1 mutation causes dyskeratosis congenita. Genes Dev. 2011;25:11-6.

49. Venteicher AS, Abreu EB, Meng Z, McCann KE, Terns RM, Veenstra TD, Terns MP, Artandi SE. A human telomerase holoenzyme protein required for Cajal body localization and telomere synthesis. Science. 2009;323:644-8.

50. Keller RB, Gagne KE, Usmani GN, Asdourian GK, Williams DA, Hofmann I, Agarwal S. CTC1 Mutations in a patient with dyskeratosis congenita. Pediatr Blood Cancer. 2012;59:311-4.

51. Du HY, Pumbo E, Ivanovich J, An P, Maziarz RT, Reiss UM, et al. TERC and TERT gene mutations in patients with bone marrow failure and the significance of telomere length measurements. Blood. 2009;113:309-16.

52. Du HY, Mason PJ, Bessler M, Wilson DB. TINF2 mutations in children with severe aplastic anemia. Pediatr Blood Cancer. 2009;52:687.

53. Liang J, Yagasaki H, Kamachi Y, Hama A, Matsumoto K, Kato $\mathrm{K}$, et al. Mutations in telomerase catalytic protein in Japanese children with aplastic anemia. Haematologica. 2006;91:656-8.

54. Yamaguchi H, Calado RT, Ly H, Kajigaya S, Baerlocher GM, Chanock SJ, et al. Mutations in TERT, the gene for telomerase reverse transcriptase, in aplastic anemia. N Engl J Med. 2005; 352:1413-24.

55. Yamaguchi H, Baerlocher GM, Lansdorp PM, Chanock SJ, Nunez O, Sloand E, et al. Mutations of the human telomerase RNA gene (TERC) in aplastic anemia and myelodysplastic syndrome. Blood. 2003;102:916-8.

56. Vulliamy T, Marrone A, Dokal I, Mason PJ. Association between aplastic anaemia and mutations in telomerase RNA. Lancet. 2002;359:2168-70.

57. Tsakiri KD, Cronkhite JT, Kuan PJ, Xing C, Raghu G, Weissler $\mathrm{JC}$, et al. Adult-onset pulmonary fibrosis caused by mutations in telomerase. Proc Natl Acad Sci USA. 2007;104:7552-7.

58. Armanios MY, Chen JJ, Cogan JD, Alder JK, Ingersoll RG, Markin C, et al. Telomerase mutations in families with idiopathic pulmonary fibrosis. N Engl J Med. 2007;356:1317-26.

59. Calado RT, Regal JA, Kleiner DE, Schrump DS, Peterson NR, Pons V, et al. A spectrum of severe familial liver disorders associate with telomerase mutations. PLoS ONE. 2009;4:e 7926.

60. Ball SE, Gibson FM, Rizzo S, Tooze JA, Marsh JC, GordonSmith EC. Progressive telomere shortening in aplastic anemia. Blood. 1998;91:3582-92.

61. Lee JJ, Kook H, Chung IJ, Na JA, Park MR, Hwang TJ, et al. Telomere length changes in patients with aplastic anaemia. $\mathrm{Br}$ J Haematol. 2001;112:1025-30. 
62. Baerlocher GM, Vulto I, de Jong G, Lansdorp PM. Flow cytometry and FISH to measure the average length of telomeres (flow FISH). Nat Protoc. 2006;1:2365-76.

63. Canela A, Klatt P, Blasco MA. Telomere length analysis. Methods Mol Biol. 2007;371:45-72.

64. Alter BP, Baerlocher GM, Savage SA, Chanock SJ, Weksler BB, Willner JP, et al. Very short telomere length by flow fluorescence in situ hybridization identifies patients with dyskeratosis congenita. Blood. 2007;110:1439-47.

65. Calado RT, Yewdell WT, Wilkerson KL, Regal JA, Kajigaya S, Stratakis CA, et al. Sex hormones, acting on the TERT gene, increase telomerase activity in human primary hematopoietic cells. Blood. 2009;114:2236-43.

66. Alter BP, Gardner FH, Hall RE. Treatment of dyskeratosis congenita with granulocyte colony-stimulating factor and erythropoietin. Br J Haematol. 1997;97:309-11.

67. Giri N, Pitel PA, Green D, Alter BP. Splenic peliosis and rupture in patients with dyskeratosis congenita on androgens and granulocyte colony-stimulating factor. Br J Haematol. 2007;138:815-7.
68. Alter BP, Giri N, Savage SA, Rosenberg PS. Cancer in dyskeratosis congenita. Blood. 2009;113:6549-57.

69. de la Fuente J, Dokal I. Dyskeratosis congenita: advances in the understanding of the telomerase defect and the role of stem cell transplantation. Pediatr Transpl. 2007;11:584-94.

70. Dietz AC, Orchard PJ, Baker KS, Giller RH, Savage SA, Alter $\mathrm{BP}$, et al. Disease-specific hematopoietic cell transplantation: nonmyeloablative conditioning regimen for dyskeratosis congenita. Bone Marrow Transpl. 2011;46:98-104.

71. Nishio N, Takahashi Y, Ohashi H, Doisaki S, Muramatsu H, Hama A, Shimada A, Yagasaki H, Kojima S. Reduced-intensity conditioning for alternative donor hematopoietic stem cell transplantation in patients with dyskeratosis congenita. Pediatr Transpl. 2011;15:161-6.

72. Agarwal S, Loh YH, McLoughlin EM, Huang J, Park IH, Miller $\mathrm{JD}$, et al. Telomere elongation in induced pluripotent stem cells from dyskeratosis congenita patients. Nature. 2010;464:292-6. 